\title{
FURTHER STUDIES OF THE X-RAY STANDARD IONIZA- TION CHAMBER DIAPHRAGM SYSTEM
}

\author{
Lauriston S. Taylor and G. Singer
}

\section{ABSTRACT}

Further studies have been made on the effect of the diaphragm system upon the calibration measurements made with the large free air ionization chamber. Air absorption coefficients were obtained, and it was found necessary to use these values to correct the ionization chamber readings. Calibrations of a thimble chamber, made with and without a restricting diaphragm close to the $\mathrm{X}$-ray tube, differ by several per cent. Dependent upon the position of the thimble chamber with respect to the tube target, the calibration may vary over a range of 10 per cent. When the radiation is not restricted by a diaphragm close to the tube, there may be a difference in the radiation quality received by the thimble and air chamber, as large as 10 per cent.

\section{CONTENTS}

I. Introduction

II. Effect of position of entrant diaphragm with respect to the collector

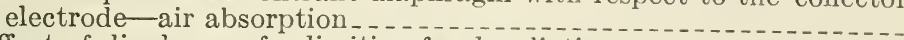

III. Effect of diaphragm for limiting focal radiation

IV. Position of thimble chamber for calibration

V. Variation of radiation quality from different parts of target and stem.-

\section{INTRODUCTION}

Since the completion of some previous studies at the bureau, of the experimental technique necessary to obtain what was believed to be a correct determination of the recently adopted unit of $\mathrm{X}$-ray quantity, several features of our own work and also that of other investigators seemed to demand further study. Certain pronounced differences appear in the results obtained by various workers, but this is perhaps to be expected, for, a careful analysis of the free air ionization chamber systems used by them leaves doubt as to whether or not the several systems should yield accordant measurements of the Röntgen.

Failla's ${ }^{1}$ thorough experimental study of the diaphragm system was carried out in a manner sufficiently different from ours to render it difficult to decide how well the respective conclusions agree. Ours ${ }^{2}$ had a limiting diaphragm close to the X-ray tube and of such sizo as to cut off all stem and off-focus radiation, whereas Failla omitted this and instead placed a somewhat larger diaphragm about $30 \mathrm{~cm}$ from the target, which, therefore, cut off only the extreme stem 
radiation. ${ }^{3}$ Again, Failla, simulating conditions used in practice, apparently obtained a satisfactory determination of the Röntgen with a system employing a single diaphragm but a different value with two diaphragms. We, however, were unable to obtain consistent results with a single diaphragm system.

Glasser since the publication of his report on the X-ray standard ionization chamber, ${ }^{4}$ informs us that he has removed the diaphragm close to the tube and finds his unit of quantity unchanged thereby, thus differing in his findings from both Failla and us. Behnken, ${ }^{5}$ using a Metalix line focus tube of the $220 \mathrm{kv}$ type, which does not demand a tube diaphragm, naturally employs a single diaphragm system.

In the present study, the effects of these different experimental conditions applied to our own system have been investigated. In addition, other geometrical arrangements of various free air ionization chamber systems have been studied.

\section{EFFECT OF POSITION OF CHAMBER DIAPHRAGM WITH RESPECT TO THE COLLECTOR ELECTRODE-AIR ABSORPTION}

From a previous analysis by Taylor of the ionization chamber diaphragm system, ${ }^{6}$ the effective ionization $E$, in the chamber, is given by

$$
E=\frac{\kappa b^{2} I_{o}}{B^{2}} L_{o}
$$

where $I_{o}$ is the $\mathrm{X}$-ray flux density at unit distance from the focus; $L_{o}$ the effective length of the measuring electrode; $B$ the distance between the tube diaphragm and the entrant diaphragm; $b$ the radius of the chamber diaphragm; and $\kappa$ a constant. This condition holds only when the aperture of the diaphragm system is filled, and absorption and scattering in the intervening air space is negligible.

When using a small focal spot tube, the focus may be considered a point (within certain ranges) so that $B$ in the relation above is replaced by $B+k$ where $k$ is the distance from focus to tube diaphragm.

Under these conditions the distance between the chamber diaphragm and collector plate should not enter. ${ }^{7}$ Consequently, we are led to expect that for a given beam of radiation, the ionization currents to the collector electrode should remain constant as this distance alone is varied, subject of course, to the one assumption that the radiation be sufficiently penetrating to permit the loss by air absorption between tube diaphragm and collector electrode to be neglected. Failla ${ }^{8}$ did find however a decrease of some 5 per cent in the ionization currents as the distance from his chamber diaphragm to collector plate was increased from 40 to $110 \mathrm{~cm}$.

He attributed most of this 5 per cent change to scattering from the lead walls of the tube which supported the diaphragms although later

\footnotetext{
"In this paper the term "stem radiation" includes that from the back of the target as well as the stem, unless stated otherwise.

( Hlasser and V. V. Portmann, Am. J. Roent., 19, p. 564; 1928.

6 II. Behnken and R. Jaeger, Strahlentherapie, 36, p. 778; 1930

I.. S. Taylor, B. S. Jour. Research, 3 (RP119), p. 807; 1929.

7 'This conclusion is in agreement with Behnken's earlier analysis. (II. Behnken, Strahlentherapie, 26, p. 70; 1927), but in discord with the work of Kaye and Binks (G. W. C. Kaye and W. Binks, Brit. J. Rad., 2, p. $553 ; 19293$

Seo p. 61, Failla's paper, referred to in footnote 1, p. 219.
} 
work on his and our part indicates that the effect was probably largely due to air absorption. ${ }^{9}$

A rough test of this eflect, made at the time our free air ionization chamber was designed, ${ }^{10}$ showed a negligible change in the ionization current when the distance between chamber diaphragm and collector plate was kept within a working range suitable to our apparatus of 30 to $45 \mathrm{~cm}$. Where the collector to chamber diaphragm distance runs to magnitudes much above this, as in Failla's experimental case, the consequent air absorption should be taken into account as shown below. To test the effect of air absorption more carefully, it was decided to repeat these measurements over a wider range of distances between chamber diaphragm and collector and, in addition, for several radiation qualities.

For this purpose the ionization chamber diaphragm, consisting of both the limiting $(N)$ and the scattering $(S)$ diaphragm, was removed from the chamber and supported rigidly in the center of the beam as indicated in Figure 1. The size of the diaphragm $N$ and its distance

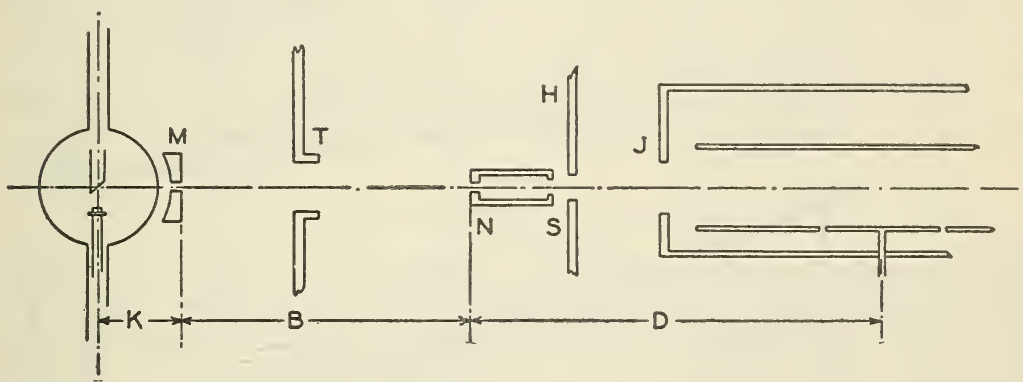

FIGURE 1.-Diagram of arrangement of ionization chamber and diaphragms used in studying air absorption

from the tube diaphragm $M$ were so adjusted that the diameter of the beam was small. The opening $J$ in the front of the chamber was about $8 \mathrm{~cm}$ in diameter, while the maximum diameter of the beam was about $3 \mathrm{~cm}$, so that, proper alignment having been assured, no part of the beam was cut off. These features were each checked by means of a fluorescent screen. Finally a lead screen $H$ was placed so as to prevent scattered radiation, from any preceding parts of the system, entering the chamber.

Maintaining $B$ constant, the ionization chamber was moved along a track, in the direction of the beam, through a variable range from $D=41.7 \mathrm{~cm}$ up. All other factories remaining constant, the resulting ionization currents in the chamber were measured by a null method, ${ }^{10}$ precautions being taken to insure that the $\mathrm{X}$-ray beam did not pass so near to the electrode as to exclude utilizing the full range of the photoelectrons.

- Doctor Failla has very kindly made certain of his unpublished data available to the authors. It is very gratifying to find that his measurements check ours as to the effect of air absorption.

${ }_{10}$ L. S. Taylor, B. S. Jour. Research, 2 (RP56), D. 771; 1929. 
TABLE 1

\begin{tabular}{|c|c|c|c|c|c|c|c|c|c|}
\hline \multirow{2}{*}{\multicolumn{2}{|c|}{ No. Tube }} & $\mathrm{kV}$ & Filter & $\underset{B}{\text { Distance }}$ & $\begin{array}{c}I_{\max } \\
D=41.7\end{array}$ & $\begin{array}{c}I_{\min } \\
D=101.7\end{array}$ & $\begin{array}{l}\text { Differ- } \\
\text { ence }\end{array}$ & $\begin{array}{c}\text { Average } \\
\text { devia- } \\
\text { tion from } \\
\text { mean }\end{array}$ & $\begin{array}{c}\mu \\
\mathrm{cm}^{-1} \\
\mathrm{X} 10^{-5}\end{array}$ \\
\hline & & 1 & 2 & 3 & 4 & 5 & $6^{\circ}$ & $\gamma$ & 8 \\
\hline (n) & $\begin{array}{l}\mathrm{A} \\
\mathrm{A} \\
\mathrm{A} \\
\mathrm{B} \\
\mathrm{B} \\
\mathrm{B} \\
\mathrm{B} \\
\mathrm{B}\end{array}$ & $\begin{array}{l}140 \\
140 \\
140 \\
133 \\
133 \\
133 \\
133 \\
112\end{array}$ & $\begin{array}{r}m m C u \\
0.00 \\
.25 \\
1.00 \\
.00 \\
.10 \\
.25 \\
1.00 \\
.25\end{array}$ & $\begin{array}{l}62.2 \\
62.2 \\
62.2 \\
40.5 \\
40.5 \\
40.5 \\
40.5 \\
40.5\end{array}$ & $\begin{array}{l}73.1 \\
38.8 \\
19.55 \\
63.69 \\
63.41 \\
75.03 \\
26.11 \\
42.37\end{array}$ & $\begin{array}{l}68.8 \\
38.0 \\
19.17 \\
60.58 \\
61.62 \\
73.03 \\
25.69 \\
41.26\end{array}$ & $\begin{array}{r}\text { Per cent } \\
6.00 \\
2.08 \\
1.96 \\
3.40 \\
2.86 \\
2.66 \\
1.65 \\
2.63\end{array}$ & $\begin{array}{r}\text { Per cent } \\
0.26 \\
.51 \\
.31 \\
.28 \\
.30 \\
.46 \\
.36 \\
-.--\end{array}$ & $\begin{array}{l}99^{*} \\
35 \\
33 \\
84^{*} \\
46 \\
44 \\
27 \\
45\end{array}$ \\
\hline
\end{tabular}

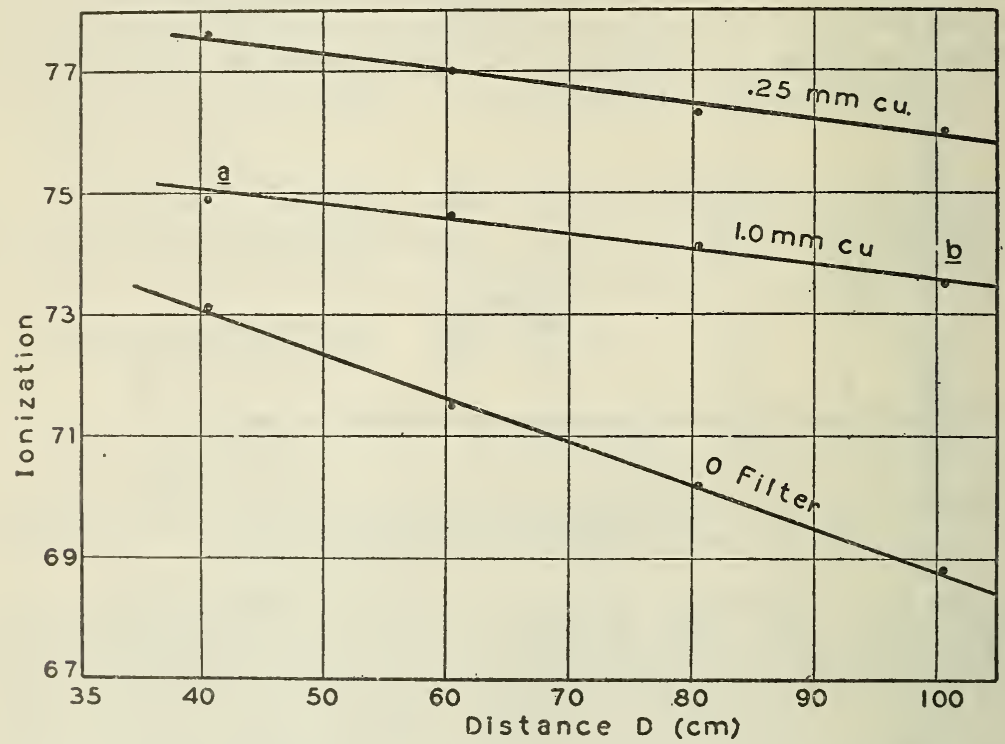

Figure 2.-Intensity of ionization as a function of the distance $D$

Figure 2 shows the plot of a typical set of such measurements, from which it is seen that the intensity of the beam falls off several per cent as the distance $D$ is increased. It is also seen that as the radiation is hardened by increasing the filtration, the absorption per unit distance becomes less.

Table 1 gives the results of several sets of such measurements. Columns 4 and 5 indicate, respectively, the maximum and minimum ionization currents obtained at $D=41.7 \mathrm{~cm}$ and $D=101.7 \mathrm{~cm}$, represented by $(a)$ and $(b)$ in Figure 2 . The percentage change in the measured ionization current appears in column 6 and the average deviation from the mean of the observations for a single run, in column 7.

The change in ionization current with increasing $D$, which is relatively large for unfiltered radiation and only one-third as much for filtered radiation, indicates that the assumption of no loss by absorption along the path certainly does not appear justified. 
From these measurements it is possible to calculate by means of the usual absorption formula, $I=I_{0} e^{-\mu x}$, fairly accurate values of the air absorption coefficients for the various qualities of radiation used. For the thickness $x$ of the absorbing medium (air) we may use the difference between the two values of $D$ at which the intensity measurements in Table 1 were made. The initial intensity $I$ is taken as that for $D=41.7 \mathrm{~cm}$ and the transmitted intensity through $60 \mathrm{~cm}$ of air as that for $D=101.7 \mathrm{~cm}$. Substitution of these values of $I$ and $I_{0}$ in the equation gives, for each radiation quality, the absorption coefficients shown in column 8 .

It should be pointed out that for very accurate determination of air absorption coefficients, it is undesirable to use such a great thickness of the absorbing medium, since there will be a slight change in the radiation quality due to absorption between the two positions of the ionization chamber used. This is, of course, more important for unfiltered radiation and, consequently, the values of $\mu$ marked with an asterisk (*) in Table 1 should not be given as much weight as the other values. In the case of filtered radiation, this error may be neglected as indicated by the fact that as the filtration of $140 \mathrm{kv}$ radiation is increased from $0.25 \mathrm{~mm}$ to $1.0 \mathrm{~mm}$ of copper, the absorption coefficient decreases from 0.00035 to $0.00033 \mathrm{~cm}^{-1}$ only.

As known, the total absorption coefficient, $\mu$, consists of two parts $\sigma$ and $\tau$ such that ${ }^{12}$

$$
\mu=\sigma+\tau
$$

where $\tau$ is the true absorption coefficient and $\sigma$ is the scattering absorption coefficient. If now $\sigma$ is appreciable, the effective ionization per cubic centimeter in the ionization chamber should be perceptibly decreased by enlarging the cross section of the beam in the chamber, either by changing the opening in the entrant diaphragm or by changing the distance $D$ in either case, the intensity being kept constant. In an earlier contribution, using the same set-up, no such change was found by the authors, ${ }^{13}$ hence we may assume that $\sigma$ plays no appreciable part here in decreasing the ionization observed.

In seeking to compare these results with previous work we find that reliable air absorption coefficients are not available. However, the use of a value of $\mu$ for air given by Eve and Day ${ }^{14}$ as $0.0004 \mathrm{~cm}^{-1}$ for roughly the same radiation quality corresponding to $112 \mathrm{kv}$ radiation filtered with $0.25 \mathrm{~mm}$ of copper, results in a decrease in intensity of about 2.4 per cent per $60 \mathrm{~cm}$ as against 2.63 per cent found in our experiments. Considering the uncertainties in the earlier work this may be taken as fair agreement.

Having found that air absorption can not be neglected we should note the effect of this on the measurements made with large free-air ionization chambers. In the chambers used by several observers the distance between chamber diaphragm and the collector plate varies from about 30 to $50 \mathrm{~cm}$. Thus, if comparing two such chambers in which this difference is appreciably different, full allowance should be made for the air absorption of each quality of radiation employed in the comparison.

\footnotetext{
12 See A. H. Compten, "X-rays and Electrons," p. 175.

13 L. S. Taylor, B. S. Jour. Research 3 (R P119), p. 807; 1929

14 A. S. Eve and F. H. Day, Phil. Mag., 23 p. 683; 1912.
} 
When calibrating a thimble chamber against a free-air chamber, the former is usually placed at the position of the free-air chamber diaphragm. Consequently the radiation, being partially absorbed within the large chamber, is more intense at the position of the small chamber than indicated by the measurements made with the large chamber. This may be corrected, by allowing for the air absorption in the air path between the chamber diaphragm and collector.

TABLE 2

\begin{tabular}{|c|c|c|c|c|c|}
\hline \multirow{2}{*}{$\mathrm{kv}$} & \multirow[t]{2}{*}{ Filter } & \multirow[t]{2}{*}{$r / \min$} & \multirow{2}{*}{$\begin{array}{l}r / \text { full } \\
\text { scale } \\
M \text { in }\end{array}$} & \multirow{2}{*}{$\begin{array}{l}r / \text { full } \\
\text { scale } \\
M \text { out }\end{array}$} & $\begin{array}{l}\text { Difference }= \\
M \text { in }-M \text { out }\end{array}$ \\
\hline & & & & & $M$ out \\
\hline 1 & 2 & 3 & 4 & 5 & 6 \\
\hline 103 & $\begin{array}{r}m m A l \\
5\end{array}$ & $\begin{array}{l}\text { 1. } 65 \\
1.76\end{array}$ & 3.02 & 2 & $\begin{array}{l}\text { Per cent } \\
\quad+1.03\end{array}$ \\
\hline 102 & 3 & $\begin{array}{l}1.54 \\
1.79\end{array}$ & 3.17 & 3.04 & +4.27 \\
\hline 85 & 0 & $\begin{array}{r}9.35 \\
11.93\end{array}$ & 3.18 & 3. 10 & +2.58 \\
\hline 108 & 5 & $\begin{array}{l}1.56 \\
1.63\end{array}$ & 2. 98 & 2.90 & +2.76 \\
\hline
\end{tabular}

In the guarded field ionization chamber, recently described by the authors, ${ }^{15}$ the distance between entrant diaphragm and collector is about $10 \mathrm{~cm}$, consequently the error due to air absorption is reduced to about 0.4 per cent for filtered radiation. For the most precise work, however, this effect should be taken into account.

\section{EFFECT OF DIAPHRAGM FOR LIMITING FOCAL RADI- ATION}

The effect of omitting the diaphragm $M$ placed next to the tube to eliminate stem and off-focus radiation was studied. Results were obtained most conveniently by comparing the ionization currents in two different chambers, both with and without the diaphragm in place. ${ }^{16}$

The method of observation was, first, with the diaphragm $M$ in place, to measure the intensity of the beam, with the large air ionization chamber and then with a thimble chamber placed at the position of the chamber entrant diaphragm; and, second, with the diaphragm $M$ removed, to repeat these measurements. In the first case the stem radiation is cut off; under the second set of conditions, to simulate practice, the beam is limited by the diaphragm $T$ having a diameter of about $4 \mathrm{~cm}$ so that a portion of the stem radiation falls on the thimble chamber or entrant diaphragm of the standard chamber, both at 90 cm from the target.

Table 2 gives the results of a series of such measurements which happened to be made at lower voltages. Similar runs for higher voltages gave essentially the same results. Column 3 gives the

13 I. S. Taylor and (1. Singer, B. S. Jour. Research, 5 (R P211), p. 507; 1930.

10 () bvinusly the magnitude of the difference betwcen the ionization currents will depend in a large measure upon the particular choice of the chambers, and consequently such results have no quantitative value other than indicating the magnitude and direction of error. 
Röntgens per minute as measured by the air chamber. Columns 4 and 5 indicate, respectively, the number of Röntgens per full scale for the thimble chamber electroscope, with $M$ in and out. Column 6 gives the percentage difference between the calibrations of the thimble chamber in the two cases.

It is seen that as might be expected when the tube diaphragm $M$ is omitted, the thimble chamber indications are in all cases larger than those of the air chamber, due to the fact that the former receives $\mathrm{X}$ rays from a greater portion of the radiating source. Thus for calibration purposes it is obviously necessary that buth chambers receive radiation from the same parts of the target.

The magnitude of such a variation depends upon the size and shape of the thimble chamber used and upon its distance from the target or diaphragm $T$. Since thimble chambers all vary in construction, it is obviously unsafe to rely upon a calibration effected by a system which does not strictly limit the beam to focal radiation.

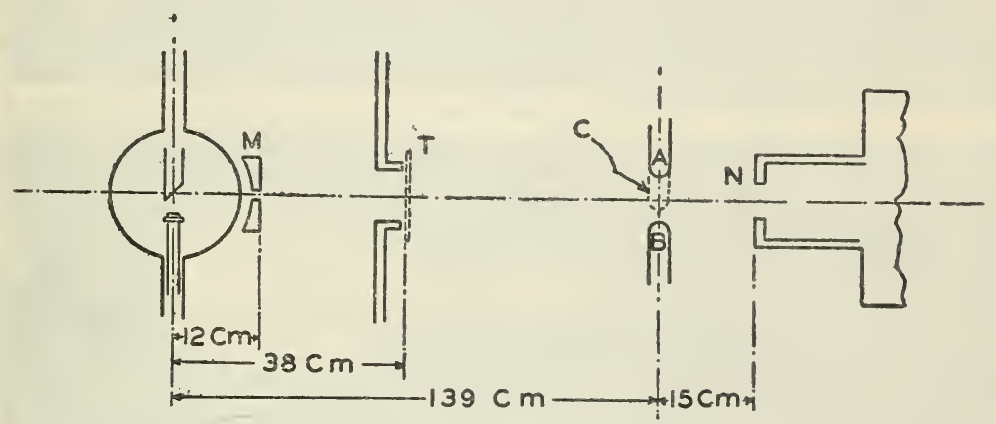

FIGURE 3.-Diagram showing arrangement of thimble chambers for calibration

\section{POSITION OF THIMBLE CHAMBER FOR CALIBRATION}

As pointed out in a previous paper by the authors, different observers employ different methods of calibrating a thimble chamber against a standard. ${ }^{17}$ It was further shown that the replacement method was the most reliable. In view of the results of Section III, it was of interest to compare the three more common methods with the additional variable factor of changing the diaphragm $M$.

Table 3

\begin{tabular}{|c|c|c|c|c|c|c|c|c|c|}
\hline \multirow{2}{*}{ No. } & \multirow{2}{*}{$\begin{array}{l}\text { Posi- } \\
\text { tion }\end{array}$} & \multirow{2}{*}{$\mathrm{k} \nabla$} & \multirow{2}{*}{ Filter } & \multirow{2}{*}{$\begin{array}{l}T / \mathrm{min} \\
M \text { in }\end{array}$} & \multirow{2}{*}{$\begin{array}{l}r / \min \\
M \text { out }\end{array}$} & \multirow{2}{*}{$\begin{array}{l}r / \text { full } \\
\text { scale } \\
M / \text { in }\end{array}$} & \multirow{2}{*}{$\begin{array}{c}r / \text { full } \\
\text { scale } \\
M \text { out }\end{array}$} & $\begin{array}{c}\text { Difference } \\
M \text { in }-M \text { out }\end{array}$ & \multirow{2}{*}{$\begin{array}{l}\text { Error of } \\
\text { observa- } \\
\text { tion }\end{array}$} \\
\hline & & & & & & & & $M$ out & \\
\hline 1 & 2 & 3 & 4 & 5 & 6 & 7 & 8 & 9 & 10 \\
\hline & $\begin{array}{l}\mathrm{A} \\
\mathrm{A} \\
\mathrm{B} \\
\mathrm{B} \\
\mathrm{C} \\
\mathrm{C}\end{array}$ & $\begin{array}{l}140 \\
140 \\
140 \\
140 \\
140 \\
140\end{array}$ & $\begin{array}{r}0.25 \\
.10 \\
.25 \\
.10 \\
.25 \\
.10\end{array}$ & $\begin{array}{l}1.396 \\
\text { 2. } 354 \\
1.420 \\
\text { 2. } 330 \\
\text { 2. } 506 \\
\text { 3. } 205\end{array}$ & $\begin{array}{l}1.418 \\
\text { 2. } 528 \\
\text { 1. } 490 \\
\text { 2. } 410 \\
\text { 2. } 668 \\
\text {. } 352\end{array}$ & $\begin{array}{l}1.412 \\
1.509 \\
1.310 \\
1.386 \\
1.429 \\
1.357\end{array}$ & $\begin{array}{l}1.501 \\
\text { 1. } 619 \\
1.285 \\
1.363 \\
1.413 \\
1.343\end{array}$ & $\begin{array}{r}\text { Per cent } \\
-5.9 \\
-6.8 \\
+1.9 \\
+1.7 \\
+1.2 \\
+1.5\end{array}$ & $\begin{array}{r}\text { Per cent } \\
0.23 \\
.27 \\
.82 \\
.48 \\
.58 \\
.53\end{array}$ \\
\hline
\end{tabular}

${ }^{17}$ L. S. Taylor and G. Singer, B. S. Jour. Research, 4 (RP169), p. 631; 1930. 
The experimental arrangement is indicated in Figure 3. A paraffin thimble chamber about $3 \mathrm{~cm}$ long was placed in three positions: (a) to one side of the beam in the direction of the tube anode, $(b)$ to the cathode side of the beam, and $(c)$ in beam center, and for each position was calibrated in Section III against the large air-chamber system with and without the diaphragm $M$ in place. The thimble chamber and air chamber were placed $139 \mathrm{~cm}$. from the target for position $c$, but the air chamber was placed $154 \mathrm{~cm}$ away for positions $a$ and $b$. In each case the alignment was tested with a fluorescent screen.

Columns 5 and 6 in Table 3 give the beam intensity in Röntgens per minute as measured with the large air chamber with diaphragm $M$ in and out, respectively. Columns 7 and 8 give the corresponding Röntgens per full scale as measured with the thimble chamber. (The electroscope used in these measurements differed from that used in Section III and had greater sensitivity.) Column 9 indicates the percentage change in the ionization measured with the thimble chamber when the diaphragm $M$ is removed. Rows 3 to 6 are seen to be in good agreement in this respect with the results given in Table 2 and further indicate that there is no essential difference in the calibration effected at positions in the center and at the cathode side of the X-ray beam. For both positions $B$ and $C$, the omission of the diaphragm $M$ raises the thimble chamber measurements about 2 per cent throwing the calibration in error by that amount.

However, rows 1 and 2 for the thimble chamber in the anode side of the beam show that there is a very large decrease in the relative ionization measured when $M$ is removed. The geometry of the system makes the reason clear; the diaphragm $T$ shields the thimble chamber from a considerable portion of the stem radiation which is allowed to enter the large air chamber. We are thus led to the conclusion that, even without the diaphragm $M$, the most satisfactory position for the calibration of a thimble chamber is in the beam center, thus necessitating a replacement method of measurement.

\section{VARIATION OF RADIATION QUALITY FROM.DIFFERENT PARTS OF TARGET AND STEM}

E. Lorenz ${ }^{18}$ found that the continuous spectrum radiation, produced by electrons striking the back of the target and stem after reflection from the target face, had a definite short wave length limit depending upon the position along the anode at which it was produced. This leads at once to the conclusion that the quality of the radiation from various parts of the anode other than the focal spot should vary over wide limits. Since the ionization measured by most small chambers depends upon the radiation quality, it is important to know to what extent such a variation in quality exists.

To determine the magnitude of such quality change, three sets of conditions were chosen by shielding off certain parts of the radiation: (a) $\Lambda$ thick lead wedge was so placed as to prevent all focal radiation from entering the air ionization chamber; $(b)$ The wedge was removed, thus allowing the total radiation from focus, target back, and stem to nnter the chamber; (c) The diaphragm $M$ (fig. 3 ) was placed in the

E. Lorenz, Proc. Nat. Acad Sci, 1f, p. 582; 1928. 
B. S. Journal of Research, RP271

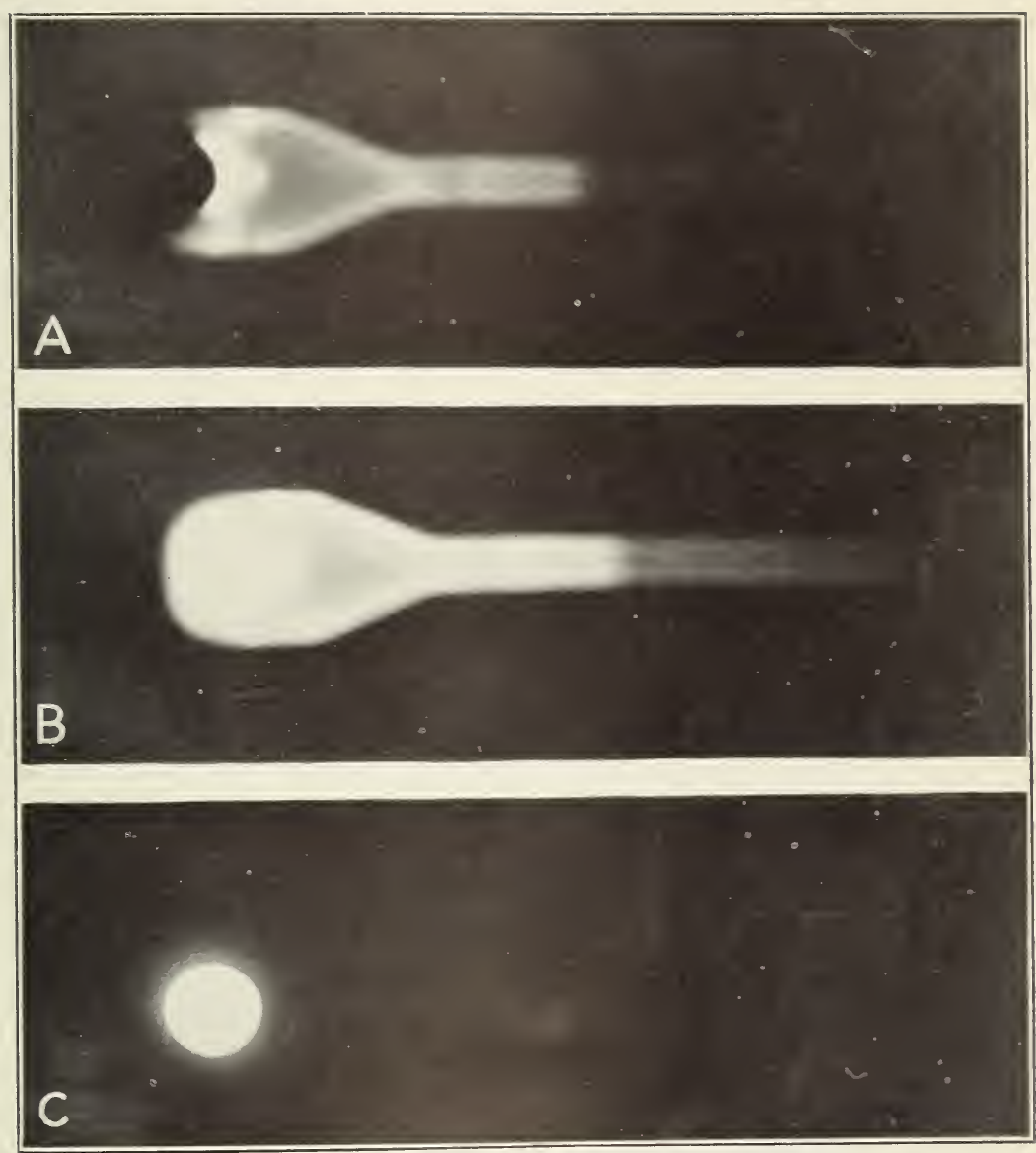

Figure 4.-Pinhole photographs of target (pinhole at position of diaphragm $T$ in fig. 1)

$A$, Tedge placed to cut off focal radiation; $B$, total radiation from anode; $C$, focal radiation only; as limited by diaphragm . .I. 
beam and adjusted to such a size that only focal radiation entered the chamber. Figure 4 shows pinhole photographs of the target for each of the three conditions. Under each set of conditions the effective wave length was determined by the finite filter method ${ }^{19}$ using a copper filter $0.05 \mathrm{~mm}$ thick. (Since all eflective wave lengths were measured by the same method there is no necessity for comparative purposes of reducing them to true effective wave lengths.)

The results of such quality measurements made with two $\mathrm{X}$-ray tubes are shown in Table 4 . A voltage of $130 \mathrm{kv}$ ripp!e potential, ${ }^{20}$ having very small ripplage, was applied to the tube. Intensity measurements $I$ were made with the free air ionization chamber for the different filtrations indicated in column 1. The transmissions of $0.05 \mathrm{~mm}$ copper for increasing initial filtration of copper are given in columns 3 and 7 . In columns 4 and 8 , and 5 and 9 , are given the corresponding copper absorption coefficients and effective wave lengths.

Comparing the quality of the focal $(C)$ and stem radiation $(A)$ for the same filtration we find a marked variation between the effective wave lengths $\lambda_{e}$ over the whole range studied. Column 10 gives the percentage difference in $\lambda_{e}$ for the two beams. Likewise there is a large difference in quality between the stem $\left(A^{\prime}\right)$ and the total radiation $(B)$. (Data $A^{\prime}$ and $B$ were for a fine focus tube while $A$ and $C$ were for a broad focus tube of the same type, so that the two are not directly comparable.) However, it is seen that the change in $\lambda_{e}$ for total $(B)$ and stem $\left(A^{\prime}\right)$ radiation is intermediate between zero change and that found for focal $(C)$ and stem $(A)$ radiation. This is to be expected, for in data $B$ we have simply an addition of a radiation of different quality to that corresponding to data $A^{\prime}$.

Furthermore the distribution of scattered electrons over the back of the target and stem will vary both with filament current and tube potential; and, as a result, there will be a variable quality for the stem radiation dependent upon these factors. In general, this will affect seriously the calibration of a thimble chamber.

These results again emphasize the importance of limiting the standard X-ray beam to the focal radiation for which the quality is uniform unless all chambers receire radiation from exactly the same portions of the anode-an obviously impractical restriction.

It has been argued that, for purposes of medical application, the thimble ionization chamber should be calibrated under the same conditions as it is used in practice; that is, exposed to the total radiation from the anode. This and previous studies by the authors show that such a method is impracticable if we are to measure X-ray intensity in terms of the international Röntgen. Moreover no errors will be introduced if the chamber is calibrated under experimentally ideal conditions and used in practice under very different conditions provided care is taken to use the proper radiation quality. If a correct calibration is in Röntgens under well-defined conditions the thimble chamber will always indicate Röntgens for beams of the same quality under which the calibration is effected. For example, if a calibration be made for a quality $\lambda_{e}$ where all the radiation comes from the focus, the chamber will indicate correctly for the same radiation quality $\lambda_{e}$ regardless of whether the source includes the stem as

10 I. S. Taylor, B. S. Jour. Research, 5 (RP212), p. 517; 1930.

20 L. S. Taylor, B. S. Jour. Research, 5 (RP 217) p. 609; 1930. 
well as the focus. The effects of variations in the radiation quality due to the source are taken care of by the very nature of the primary calibration of a thimble chamber.

TABLE 4

$A^{\prime}$ (stem-0. F.)

B (F. tstem +O. F.)

\begin{tabular}{|c|c|c|c|c|c|c|c|c|c|}
\hline Filter & $I$ & $I_{2} / I_{1}$ & $\mu$ & $\lambda_{0}$ & $I$ & $I_{2} / I_{1}$ & $\mu$ & $\lambda_{0}$ & $\Delta \lambda_{\theta}$ \\
\hline 1 & 3 & 3 & 4 & 5 & 6 & $y$ & 8 & 9 & 10 \\
\hline \multirow{3}{*}{$\begin{array}{r}0.10 \\
.15 \\
.20 \\
.25\end{array}$} & 5700 & & $c m-1$ & & \multirow[b]{2}{*}{$\begin{array}{l}57.90 \\
45.20 \\
38.70 \\
34.00\end{array}$} & & $c m-1$ & $A$ & Per cent \\
\hline & $\begin{array}{l}43.00 \\
35.30 \\
30.20\end{array}$ & $\begin{array}{r}0.754 \\
.821 \\
.856\end{array}$ & $\begin{array}{l}56.4 \\
39.4 \\
31.0\end{array}$ & $\begin{array}{r}0.338 \\
.296 \\
.272\end{array}$ & & $\begin{array}{r}0.780 \\
.856 \\
.878\end{array}$ & $\begin{array}{l}49.8 \\
31.0 \\
26.0\end{array}$ & $\begin{array}{r}0.322 \\
.272 \\
.255\end{array}$ & $\begin{array}{l}4.85 \\
8.45 \\
6.44\end{array}$ \\
\hline & \multicolumn{4}{|c|}{ A (Stem+O. F.) } & \multicolumn{5}{|c|}{ C (Focal) } \\
\hline \multirow[t]{7}{*}{0.10} & 62.97 & & & & 80.37 & & & & \\
\hline & 45.34 & 0.720 & 65.8 & 0.357 & 62.25 & 0.776 & 50.6 & 0.324 & 6.76 \\
\hline & 36. 26 & .801 & 44.4 & .310 & 52.90 & .847 & 33.4 & .280 & 10.16 \\
\hline & $\begin{array}{l}30.50 \\
26.40\end{array}$ & $\begin{array}{r}.841 \\
866\end{array}$ & $\begin{array}{l}34.6 \\
28.8\end{array}$ & $\begin{array}{r}.282 \\
.265\end{array}$ & 46.20 & .873 & 27.2 & .259 & $\begin{array}{r}8.49 \\
14.10\end{array}$ \\
\hline & 23.31 & .882 & 25. & .25 & & .910 & & .225 & 11. 70 \\
\hline & 20.86 & .894 & 22.4 & .241 & 34. & .913 & & .222 & 8. 19 \\
\hline & 18.46 & .884 & 22.6 & .242 & 31.94 & .914 & 18.0 & .221 & 9.0 \\
\hline
\end{tabular}

Washington, October 1, 1930. 